\title{
Gold Nanoparticle-Coated Microspheres for Enhancing Immunosensor Detection of Hepatitis B Virus Surface Antibody
}

\author{
Pitirat Pholpabu ${ }^{1, *}$, Rungtiva P. Poo-arporn', Dujduan Waraho-Zhmayev ${ }^{1}$, Boonnisa Watcharapathorn ${ }^{2}$, \\ Chanikan Thongdaeng ${ }^{2}$, Paisit Luesiripanich ${ }^{2}$, Thonthun Tueanwiradet ${ }^{2}$, Jadsadakorn Juntratip ${ }^{2}$ \\ ${ }^{1}$ Biological Engineering Program, Faculty of Engineering King Mongkut's University of Technology Thonburi \\ Bangkok, Thailand \\ pitirat.pho@mail.kmutt.ac.th \\ ${ }^{2}$ Darunsikkhalai Science School \\ King Mongkut's University of Technology Thonburi \\ Bangkok, Thailand
}

\begin{abstract}
Gold nanoparticle-coated calcium carbonate microspheres (NPCM) were fabricated and utilized to create a paper-based lateral flow immunosensor with an enhanced limit of detection of hepatitis B virus surface antibody. Spherical calcium carbonate microspheres in a form of vaterite crystal were successfully synthesized and coated with gold nanoparticles to serve as a signaling material, as opposed to typical free gold nanoparticles (AuNP). The size of calcium carbonate microspheres was controlled by an addition of ethylene glycol and dropwise precipitation to facilitate a flow of NPCM through a nitrocellulose membrane. The results demonstrate that the colorimetric signal of HBsAb detection was enhanced by NPCM, resulting in the lower limit of detection from $100 \mathrm{ng} / \mathrm{mL}$, when using AuNP, to as low as $20 \mathrm{ng} / \mathrm{mL}$. Determination of positive/negative result was in only 20 min. Gold nanoparticle-coated microspherebased immunosensor should be, consequently, considered as an efficient signaling material for designing a paper-based lateral flow immunosensor for a detection of biomolecules.
\end{abstract}

Keywords: test strip, gold nanoparticle, calcium carbonate microsphere, lateral flow, immunosensor

\section{Introduction}

Hepatitis B virus infection is one of the major causes of worldwide liver cancers that threaten more than 650,000 patient lives out of 240 million who get infected annually [1]. The widespread of the infection results in a great demand of screening and testing devices to determine the infection stage as well as the patient immunity. Detection of hepatitis B virus surface antibody is part of a blood test that verifies an occurrence of hepatitis B immunity in patients who has been recovered from the infection or vaccinated. That means the patient has no infection and can no longer spread the disease to others.

Paper-based lateral flow immunosensor is a diagnostic device that is based on principle of specific bindings of antigens and antibodies under a horizontal flow of detecting materials and analytes. The sensor is considered fast, cheap, no requirement of professionalship to complete a test, and can be easily used for a point-of-care testing. However, most of the sensors still have some challenges on low analytical sensitivity with poor limit of detection [2].

Calcium carbonate microspheres (CM) are a result of crystal formation in a certain condition to obtain a specific crystal form of vaterite; not aragonite or calcite [3]. Vaterites are spherical and constructed of porous structure that is very useful for encapsulating small molecules and nanoparticles, especially for biomedical applications because of its biocompatibility [4]-[6]. In this study, we coated CM with gold nanoparticles to create an efficient colorimetric signaling material for a paperbased lateral flow immunosensor for HBsAb detection. Limit of detection of the sensor with NPCM as a signaling material was compared to the one with AuNP. 


\section{Materials and Methods}

\subsection{Chemicals and Materials}

Calcium chloride $\left(\mathrm{CaCl}_{2}\right)$, sodium carbonate $\left(\mathrm{Na}_{2} \mathrm{CO}_{3}\right)$, and phosphate-buffer saline (PBS, $0.01 \mathrm{M}$, pH 7.4), ethylene glycol (99\%), gold nanoparticles (AuNP, $40 \mathrm{~nm}$ in diameter), and DI water were purchased from Alfa Aesar. Ethanol was purchased from Apex Alco. Mouse anti-hepatitis B virus surface antigen antibody (HBsAb) was purchased from Fitzgerald. Hepatitis B virus surface antigen (HBsAg) was purchased from Prospec. Rabbit anti-mouse IgG (Rb anti-Ms) and Mouse anti-rabbit IgG (Ms anti-Rb) were purchased from Abcam. Sucrose was purchased from Sigma-Aldrich. Bovine Serum Albumin (BSA) and Tween20 were purchased from Bio Basic Canada Inc. All chemicals were used as received without further purification. Nitrocellulose membrane (UniSart CN95) was purchased from Sartorius. Cellulose fiber (Whatman Cellulose 470) and glass fiber (GF33) were purchased from GE Healthcare Life Sciences.

\subsection{Preparation of Calcium Carbonate Microspheres (CM)}

$\mathrm{CM}$ were prepared using two different methods to generate two different sizes of the microspheres. The first method was performed according to our previous report [4]. Briefly, $\mathrm{CaCl}_{2}$ and $\mathrm{Na}_{2} \mathrm{CO}_{3}$ aqueous solutions with equal molar of 0.33 $\mathrm{M}$ and volume of $50 \mathrm{~mL}$ were separately prepared and rapidly mixed together. The saturated mixture was allowed to precipitate for $20 \mathrm{~min}$ at room temperature and collected by centrifugation at 2,000 $\mathrm{g}$ for $1 \mathrm{~min}$. CM were then washed with 95\% ethanol three times and dried in vacuum. To reduce the size of CM, dropwise precipitation was performed in a presence of ethylene glycol [7]. Initially, ethylene glycol was mixed with water in a ratio of 2:1 by volume to be used as a solvent to prepare both $\mathrm{CaCl}_{2}$ and $\mathrm{Na}_{2} \mathrm{CO}_{3}$ solutions. After that, instead of rapid mixing, the $\mathrm{CaCl}_{2}$ solution was gradually added to the $\mathrm{Na}_{2} \mathrm{CO}_{3}$ solution at a dropping rate of $0.1 \mathrm{~mL} / \mathrm{min}$ under continuous magnetic-stirring at $1200 \mathrm{rpm}, 30{ }^{\circ} \mathrm{C}$. The dried $\mathrm{CM}$ were stored at $4{ }^{\circ} \mathrm{C}$ before use. The microstructure of the $\mathrm{CM}$ was characterized using scanning electron microscope (SEM; JEOL, JSM-6610 LV) equipped with energy dispersive X-ray spectroscopy (EDS; Oxford, INCA-xart). The SEM micrographs were analyzed using NIH ImageJ (National Institute of Health, USA).

\subsection{Preparation of Antibody Conjugated Nanoparticle-Coated Microspheres (NPCM*)}

Nanoparticle coating on CM surface and antibody conjugation were subsequently performed to generate NPCM*. Initially, AuNP was adjusted to $\mathrm{pH} 7$ using $\mathrm{K}_{2} \mathrm{CO}_{3}$ and added with $\mathrm{CM}$ to a final concentration of $10 \mathrm{mg} / \mathrm{mL}$. The suspension was sonicated for $20 \mathrm{~min}$ at room temperature and centrifuged to collect NPCM at 2,000 g for $1 \mathrm{~min}$. The supernatant was then removed, and the pellet was washed with DI water three times before resuspended in $0.5 \mathrm{~mL}$ of DI water. After that, Rb anti-Ms was added to NPCM to have a final concentration of $10 \mu \mathrm{g} / \mathrm{mL}$. The suspension was then gently shaken for $1 \mathrm{~h}$ and centrifuged at 4,000 g for 30 min. The pellet was resuspended in PBS with $1 \%(\mathrm{w} / \mathrm{v}) \mathrm{BSA}$ and stored at $4{ }^{\circ} \mathrm{C}$ until use. Antibody conjugated gold nanoparticles (AuNP*) were prepared in the same manner using Rb anti-Ms as the conjugating antibody. The antibody conjugation was confirmed by absorbance spectrum scanning from 450 to $650 \mathrm{~nm}$ using microplate reader (Infinite 200 Tecan, Austria).

\subsection{Preparation and Assembly of Immunosensor Strip}

Glass fiber was immersed in PBS with $1 \%$ (v/v) TWEEN20, $2 \%$ (w/v) BSA, and $2.5 \%$ (w/v) sucrose for 30 min, dried overnight, and cut into pieces of $0.5 \times 1 \mathrm{~cm}^{2}$ to be used as sample pads. For conjugate pads, a piece of the glass fiber was further treated with $20 \mu \mathrm{L}$ of AuNP* or NPCM*, dried for $1 \mathrm{~h}$, and cut into pieces of $0.5 \times 0.5 \mathrm{~cm}^{2}$. For test and control lines, nitrocellulose membrane was coated with $0.5 \mathrm{mg} / \mathrm{mL}$ of HBsAg and $0.5 \mathrm{mg} / \mathrm{mL}$ of Ms anti-Rb, respectively, using $1 \mu \mathrm{L}$ of each per $1 \mathrm{~cm}$. The membrane was then cut into pieces of $0.5 \times 2.5 \mathrm{~cm}^{2}$. The components were finally assembled to create a test strip as shown in Fig. 3.

\subsection{Preparation and Assembly of Immunosensor Strip}

To compare the limit of detection between the device using AuNP* as a signaling material and the one enhanced with $\mathrm{NPCM}^{*}, \mathrm{HBsAb}$ was diluted to different concentrations of $100,50,20$, and $10 \mathrm{ng} / \mathrm{mL}$, and dropped on a sample pad of each test strip $(70 \mu \mathrm{L})$ to investigate the signaling color of the test and control lines. Essentially, presence of HBsAb can be 
visualized by red color on the test line, and presence of AuNP* or NPCM* can be visualized by red color on the control line. Two colored lines indicate a positive result: HBsAb is detected. Colored control line and uncolored test line indicate a negative result: $\mathrm{HBsAb}$ is undetected. Uncolored control line indicates failure of the strip.

\section{Results and Discussion}

\subsection{Characterization of Calcium Carbonate Microspheres}

SEM micrographs in Fig. 1 shows that both preparation methods yield CM in spherical vaterite crystal form. Presence of ethylene glycol and dropwise precipitation significantly reduced the diameter of CM from $7.86 \pm 1.22$ to $3.06 \pm 0.95 \mu \mathrm{m}$, compared to CM prepared with no ethylene glycol. The results correspond to the previous report that demonstrated the effect of viscosity and dropwise precipitation on the calcium carbonate crystal shape and size [7]. It is noted that both sizes of CM are much smaller than the pore size of nitrocellulose membrane $(15.0 \mu \mathrm{m})$, so CM should be able to diffuse through the device. For a membrane with smaller pore size, the percentage of ethylene glycol or dropping rate might be adjusted to obtain smaller size of CM [7]. For EDS spectra of CM, Fig. 1 c) and d) reveal individual elements, including $\mathrm{Ca}, \mathrm{C}, \mathrm{O}$, and $\mathrm{Na}$, similarly to previously reported elements of calcium carbonate nanocrystals [8]. Na was found less than $1 \%$ by weight of $\mathrm{CM}$ from both methods. The results indicate that ethylene glycol can be all removed out from the crystal by centrifugation and there is no significant difference in elemental compositions of CM fabricated from those two methods.
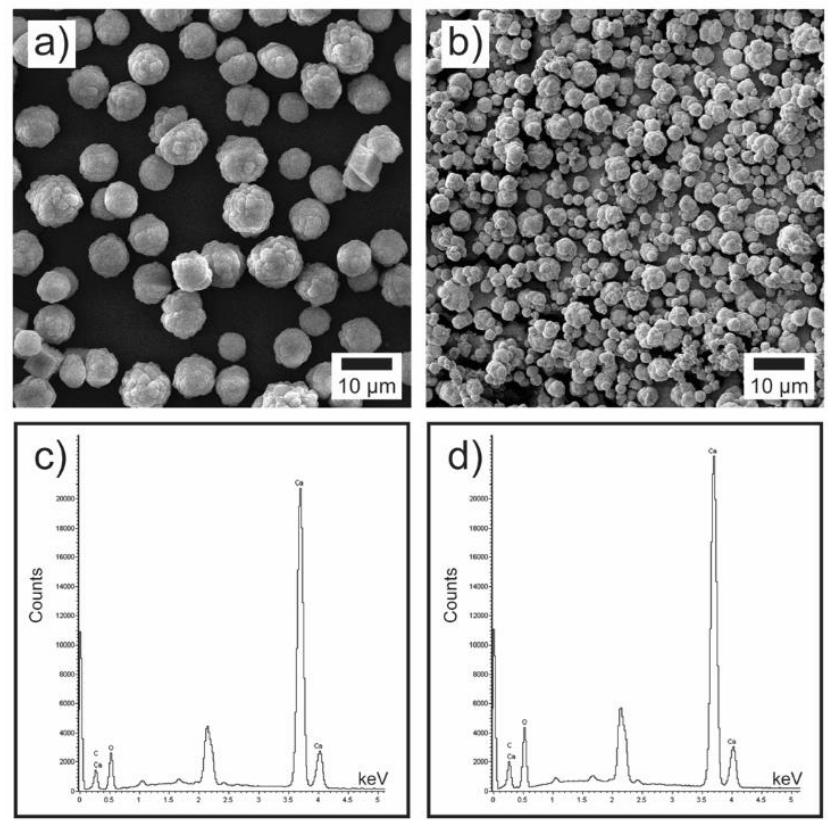

Fig. 1: SEM micrographs and EDS spectra of CM prepared by a), c) rapid mixing of saturated $\mathrm{CaCl}_{2}$ and $\mathrm{Na}_{2} \mathrm{CO}_{3}$ aqueous solutions; b), d) dropwise precipitation with a presence of ethylene glycol.

\subsection{Characterization of AuNP* and $\mathrm{NPCM}^{\star}$}

Success of antibody conjugation on the surface of AuNP and NPCM was determined by UV-VIS absorption spectrometry. As shown in Fig. 2, the absorption peak of AuNP was found at $530 \mathrm{~nm}$, and shifted to $534 \mathrm{~nm}$ when the nanoparticle was conjugated (AuNP*). The antibody conjugation has been commonly done to functionalize AuNP because of the high stability as a result of cysteine-AuNP interaction [9]. Similarly to AuNP*, the absorption peak of NPCM* was found at $534 \mathrm{~nm}$, demonstrating that the antibody was successfully absorbed on the NPCM surface that was covered by AuNP [5]. The electrostatic interaction between AuNP and CM was caused by positively charged CM, which has an isoelectric point at $\mathrm{pH} 8.5$, and negatively charged AuNP [10]. 


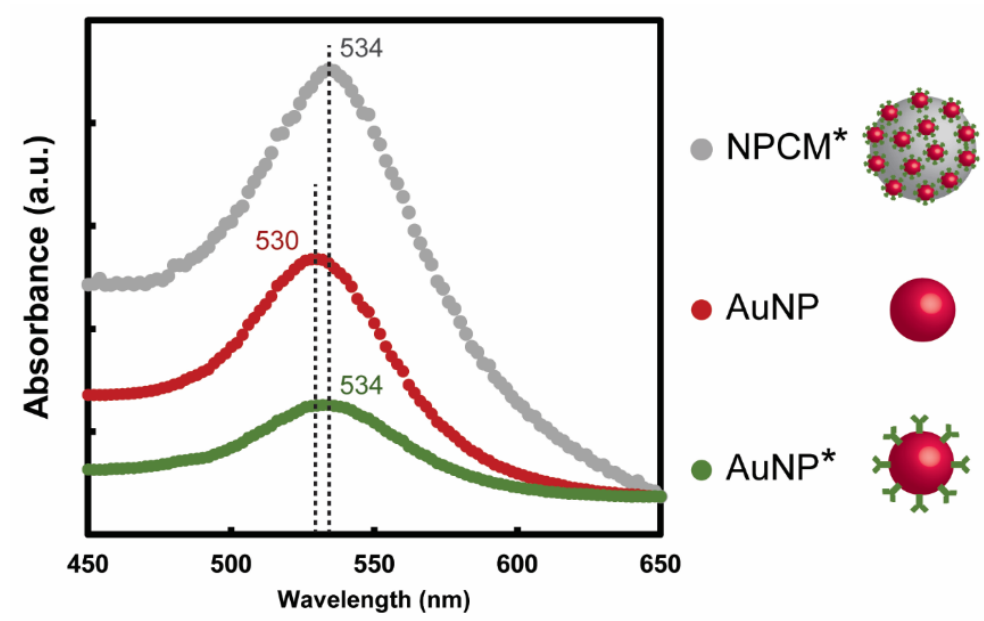

Fig. 2: UV-VIS absorption spectra of AuNP, AuNP*, and NPCM*.

\subsection{Enhanced Detection of HBsAb}

Semi-quantitative detection of HBsAb was successfully performed using the paper-based lateral-flow immunosensor, as depicted in Fig. 3. As shown in Fig. 4, red lines were shown at the control lines of all test strips except the one containing the large $\mathrm{NPCM}^{*}$, of which the diameter was about half of the pore size of the nitrocellulose membrane. Most of the NPCM* was apparently stuck at the edge between the conjugate pad and the membrane, resulting in an incomplete flow of the material. The small $\mathrm{NPCM}^{*}$, which has the diameter about one-fifth of the membrane pore size, could easily diffuse through the membrane and provided the detection results within $20 \mathrm{~min}$, similarly to AuNP*. The results suggest that the size of CM is crucial and should be much smaller than the membrane pore size.

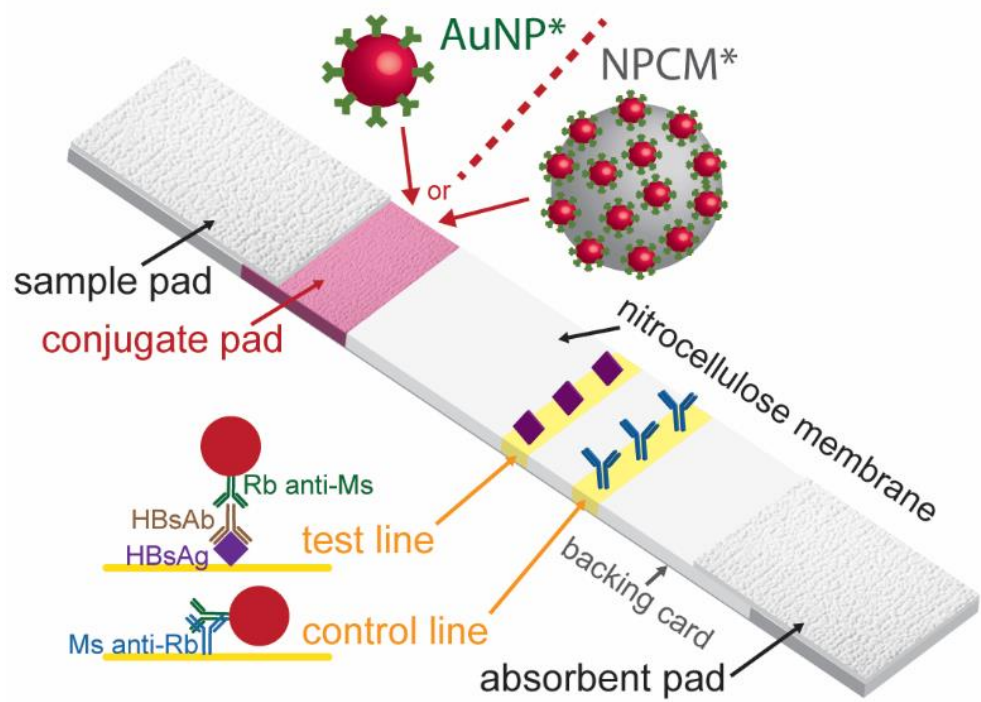

Fig. 3: Schematic of paper-based immunosensor showing the device components and detection approach. The test line provides red signaling line responding to a presence of HBsAb that specifically binds to immobilized HBsAg and is captured by AuNP*. In the meantime, the control line confirms a success of the detection process by using immobilized Ms anti-Rb that specifically binds Rb antiMs that is conjugated on the surface of AuNP* or NPCM*.

Use of NPCM* as a signaling material in the paper-based immunosensor resulted in lower limit of detection of HBsAb, compared to the one with AuNP*. That means smaller amount of HBsAb was required for showing a positive result for the 
sensor with NPCM*. As shown in Fig. 4, the positive results, two red lines, can be observed only at $100 \mathrm{ng} / \mathrm{mL}$ of $\mathrm{HBsAb}$ for the test strip with AuNP*, while, for the strip with NPCM*, HBsAb was detectable from 100 to as low as $20 \mathrm{ng} / \mathrm{mL}$. This limit of detection is also lower than a recently reported research on HBsAb detection using similar AuNP-based immunosensor [11]. Additionally, the results agree with the improved detection limit of a protein using hollow nanogold microspheres, instead of typical gold nanoparticles, in a lateral-flow test strip [5]. The number of gold nanoparticles per analyte capturing area might be the key of this enhanced detection. For AuNP*, the limit of detection was approximated to the particle diameter to the power of negative three $\left(d^{-3}\right)$, meaning the bigger AuNP* would result in less detection limit [12]. However, one analyte was captured by the limited number of AuNP*, so there was not much gold nanoparticles per area. In contrast, one $\mathrm{NPCM}^{*}$ was packed of a number of AuNP*, so the colored signal was enhanced for visualization especially when the number of analytes was minimal. The overall results suggest that NPCM can enhance the detection of HBsAb at low concentration. Since paper-based lateral-flow test strips typically use similar approach for analyte detection, this NPCM platform is greatly promising to improve the detection of various biomolecules. The improved cutoff value for determining positive/negative result will provide more precise screening of an interested analyte.

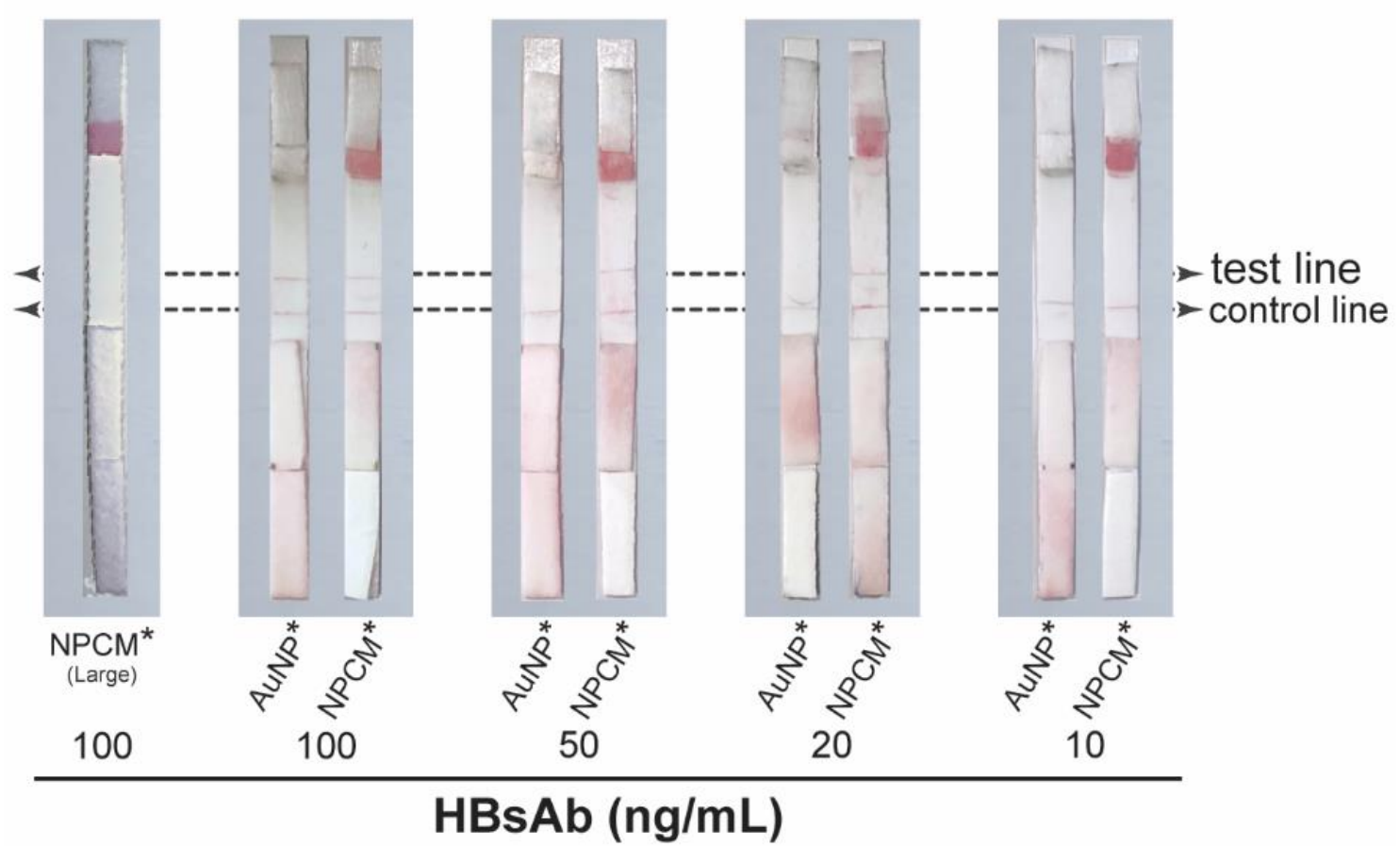

Fig. 4: Semi-quantitative results of HBsAb detection. The control line can be all visualized except the strip with large NPCM*. Positive results with two red lines occur at only $100 \mathrm{ng} / \mathrm{mL}$ for AuNP*, and at 100, 50, and $20 \mathrm{ng} / \mathrm{mL}$ for NPCM*.

\section{Conclusion}

In this study, fabrication of paper-based lateral flow immunosensor with NPCM* was successfully performed and provided lower limit of detection, compared to the AuNP-based sensor. The size of CM could be controlled using ethylene glycol and dropwise precipitation to be compatible to the pore size of the membrane. No ethylene glycol residue was observed. CM decently served as a AuNP carrier to improve the colorimetric signal especially for a diluted analyte. A positive result of detection was clearly observed at as low concentration as $20 \mathrm{ng} / \mathrm{mL}$ of HBsAb. Taken together, the gold nanoparticle-coated microspheres provide a great opportunity to re-design a paper-based lateral flow immunosensor for more accurate screening of a disease with enhanced signaling and lower limit of detection. 


\section{Acknowledgements}

The authors acknowledge funding provided by the research subsidy fund from King Mongkut's University of Technology Thonburi.

\section{References}

[1] J. M. Serigado, M. Izzy, and H. Kalia, "Novel therapies and potential therapeutic targets in the management of chronic hepatitis B:," European Journal of Gastroenterology \& Hepatology, vol. 29, no. 9, pp. 987-993, Sep. 2017, doi: 10.1097/MEG.0000000000000911.

[2] Urusov, Zherdev, and Dzantiev, "Towards Lateral Flow Quantitative Assays: Detection Approaches," Biosensors, vol. 9, no. 3, p. 89, Jul. 2019, doi: 10.3390/bios9030089.

[3] T. Ogino, T. Suzuki, and K. Sawada, "The formation and transformation mechanism of calcium carbonate in water," Geochimica et Cosmochimica Acta, vol. 51, no. 10, pp. 2757-2767, Oct. 1987, doi: 10.1016/0016-7037(87)90155-4.

[4] P. Pholpabu, P. Somharnwong, N. Huaybun, C. Cherdbaramee, V. Boonpasart, L. Komchum, and A. Phuengsap, "Controlled Release of Dual Antibacterial Drug from Composite Hydrogels," in 2019 12th Biomedical Engineering International Conference (BMEiCON), Ubon Ratchathani, Thailand, Nov. 2019, pp. 1-5, doi: 10.1109/BMEiCON47515.2019.8990291.

[5] K. Zhang, J. Wu, Y. Li, Y. Wu, T. Huang, and D. Tang, "Hollow nanogold microsphere-signalized lateral flow immunodipstick for the sensitive determination of the neurotoxin brevetoxin B," Microchim Acta, vol. 181, no. 11, pp. 1447-1454, Aug. 2014, doi: 10.1007/s00604-014-1291-9.

[6] N. Qiu, H. Yin, B. Ji, N. Klauke, A. Glidle, Y. Zhang, H. Song, L. Cai, L. Ma, G. Wang, L. Chen, and W. Wang, "Calcium carbonate microspheres as carriers for the anticancer drug camptothecin," Materials Science and Engineering: C, vol. 32, no. 8, pp. 2634-2640, Dec. 2012, doi: 10.1016/j.msec.2012.08.026.

[7] Y. I. Svenskaya, H. Fattah, O. A. Inozemtseva, A. G. Ivanova, S. N. Shtykov, D. A. Gorin, and B. V. Parakhonskiy, "Key Parameters for Size- and Shape-Controlled Synthesis of Vaterite Particles," Crystal Growth \& Design, vol. 18, no. 1, pp. 331-337, Jan. 2018, doi: 10.1021/acs.cgd.7b01328.

[8] A. Shafiu Kamba, M. Ismail, T. A. Tengku Ibrahim, and Z. A. B. Zakaria, "Synthesis and Characterisation of Calcium Carbonate Aragonite Nanocrystals from Cockle Shell Powder ( Anadara granosa )," Journal of Nanomaterials, vol. 2013, pp. 1-9, 2013, doi: 10.1155/2013/398357.

[9] J. Zong, S. L. Cobb, and N. R. Cameron, "Peptide-functionalized gold nanoparticles: versatile biomaterials for diagnostic and therapeutic applications," Biomater. Sci., vol. 5, no. 5, pp. 872-886, 2017, doi: 10.1039/C7BM00006E.

[10] W.-Y. Cai, Q. Xu, X.-N. Zhao, J.-J. Zhu, and H.-Y. Chen, "Porous Gold-Nanoparticle-CaCO3 Hybrid Material: Preparation, Characterization, and Application for Horseradish Peroxidase Assembly and Direct Electrochemistry," Chem. Mater., vol. 18, no. 2, pp. 279-284, Jan. 2006, doi: 10.1021/cm051442i.

[11] C. Akkapinyo, P. Khownarumit, D. Waraho-Zhmayev, and R. P. Poo-arporn, "Development of a multiplex immunochromatographic strip test and ultrasensitive electrochemical immunosensor for hepatitis B virus screening," Analytica Chimica Acta, vol. 1095, pp. 162-171, Jan. 2020, doi: 10.1016/j.aca.2019.10.016.

[12] B. N. Khlebtsov, R. S. Tumskiy, A. M. Burov, T. E. Pylaev, and N. G. Khlebtsov, "Quantifying the Numbers of Gold Nanoparticles in the Test Zone of Lateral Flow Immunoassay Strips," ACS Appl. Nano Mater., vol. 2, no. 8, pp. 50205028, Aug. 2019, doi: 10.1021/acsanm.9b00956. 